\title{
The electromagnetic calorimeter in JLab Real Compton Scattering Experiment
}

\author{
A. Shahinyan ${ }^{\text {a,* }}$, T.-H. Chang ${ }^{\text {b }}$, E. Chudakov ${ }^{c}$, A. Danagoulian ${ }^{\text {b }}$, P. Degtyarenko ${ }^{\text {c }}$, K. Egiyan $^{\text {a, }}{ }^{\text {, }}$, \\ V. Gorbenko ${ }^{\mathrm{d}}$, J. Hines ${ }^{\mathrm{e}, 2}$, E. Hovhannisyan ${ }^{\mathrm{a}, 1}$, Ch. Hyde $^{\mathrm{f}}$, C.W. de Jager $^{\mathrm{c}}$, A. Ketikyan ${ }^{\mathrm{a}}$, \\ V. Mamyan ${ }^{\mathrm{a}, \mathrm{c}}$, R. Michaels ${ }^{\mathrm{c}}$, A.M. Nathan ${ }^{\mathrm{b}}$, V. Nelyubin ${ }^{\mathrm{g}}$, I. Rachek ${ }^{\mathrm{h}}$, M. Roedelbrom ${ }^{\mathrm{b}}$,

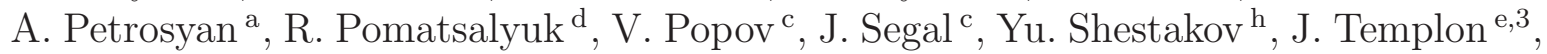 \\ H. Voskanyan ${ }^{\mathrm{a}}$, and B. Wojtsekhowski ${ }^{\mathrm{c}}$ \\ a Yerevan Physics Institute, Yerevan 375036, Armenia \\ ${ }^{\mathrm{b}}$ University of Illinois, Urbana-Champaign, IL 61801 \\ c Thomas Jefferson National Accelerator Facility, Newport News, VA 23606 \\ d Kharkov Institute of Physics and Technology, Kharkov 61108, Ukraine \\ e The University of Georgia, Athens, GA 30602, USA \\ f Old Dominion University, Norfolk, VA 23529 \\ g St. Petersburg Nuclear Physics Institute, Gatchina, 188350, Russia \\ ${ }^{\mathrm{h}}$ Budker Institute for Nuclear Physics, Novosibirsk 630090, Russia
}

\section{Abstract}

A hodoscope calorimeter comprising of 704 lead-glass blocks is described. The calorimeter was constructed for use in the JLab Real Compton Scattering experiment. The detector provides a measurement of the coordinates and the energy of scattered photons in the $\mathrm{GeV}$ energy range with resolutions of $5 \mathrm{~mm}$ and $6 \% / \sqrt{E_{\gamma}[\mathrm{GeV}]}$, respectively. Design features and performance parameters during the experiment are presented.

Key words: Calorimeters, Čerenkov detectors

PACS: $29.40 \mathrm{Vj}, 29.40 . \mathrm{Ka}$

\section{Introduction}

This calorimeter was constructed as part of the instrumentation of JLab experiment E99-114, "Exclusive Compton Scattering on the Proton" [1], of which the schematic layout is shown in Fig. 1.

The study of elastic photon scattering provides important information about nucleon structure, which is complementary to that obtained from elastic electron scattering [2]. Experimental data on the Real Compton Scattering (RCS) process at large photon energy and large scattering angle are rather scarce, mainly due to the complexity of high-

\footnotetext{
* tel: +1 7572695516

Email address: shahinya@jlab.org (A. Shahinyan).

1 deceased

2 present address: Applied Biosystems/MDS, USA

3 present address: NIKHEF, 1009 DB Amsterdam, The Netherlands
}

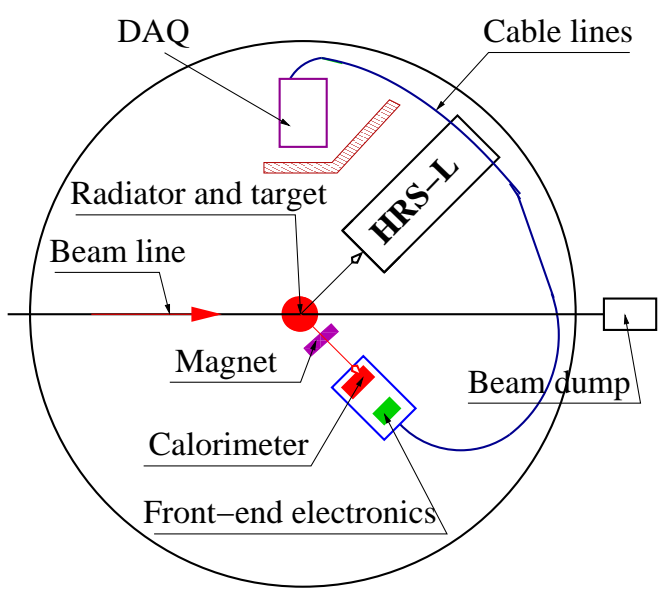

Fig. 1. Layout of the RCS experiment in Hall A. An electron beam incident on a radiator produces an intense flux of high energy photons. 
resolution photon detectors. Even the basic mechanism of the RCS reaction is the subject of active debate $[3,4,5]$. The only data available before the JLab E99-114 experiment were obtained at Cornell about 30 years ago [6]. With the construction of the CEBAF accelerator many experiments with electron and photon beams in the $\mathrm{GeV}$ energy range have been extended with much improved precision. This is the result of a number of fundamental improvements to the electron beam, including a $100 \%$ duty cycle, a low emittance and a high polarization, and new targets and detectors. The CEBAF duty factor provides an improvement of a factor of 15 compared to the best duty factor of a beam extracted from a synchrotron, at a similar instantaneous rate in the detectors.

In 1994 we started the development of a technique for an RCS experiment and in 1997, when the first proposal was approved, initiated large-scale prototyping. The results of the test runs in 1998 and 1999 [7] provided sufficient information for the final design of the apparatus presented in this article. The actual experiment was performed (see Fig. 1) at a photon-nucleon luminosity which was a factor of 1300 higher than the one in the Cornell experiment. The mixed electron-photon beam was incident on a liquid hydrogen target and passes to a beam dump. The scattered photons were detected in the calorimeter, while the recoiling protons were detected in a magnetic spectrometer HRS-L. A magnet between the hydrogen target and the calorimeter deflects the scattered electrons, which allows the selection of Compton scattering events. The Data Acquisition Electronics (DAQ) is shielded by a 4" thick concrete wall from the beam dump and the target. Figure 2 shows a picture of the experimental set-up with the calorimeter in the center. The present RCS experiment at high photon energy utilized a proton-photon time coincidence and an accurate measurement of the proton-photon kinematic correlations. The largest improvement in the rate for the JLab experiment was achieved through a mixed electron-photon beam, the application of which requires a veto detector in front of the photon detector or the magnetic deflection of the scattered electron [1]. The mixed beam approach doesn't rely on a clean photon beam and allows to locate the photon radiator very close to the hydrogen target. It also confines the background near the beam line and reduces the photon beam size. This small beam size in combination with the large dispersion in the HRS spectrometer [8] results in very good momentum and angle resolutions for the recoiling proton without having to use a tracking detector near the target, where the background rate is very high.

Good energy and coordinate resolutions are key features of the photon detector, both of which were significantly improved in the JLab experiment as compared to the Cornell one. An energy resolution of at least $10 \%$ is required to separate the RCS events from the electron bremsstrahlung events and to identify the neutral pion events. To deal with the neutral pion background, which is a dominant part of the high-energy background in the RCS experiment, a high angular resolution is crucial. It was obtained by con- structing a highly segmented calorimeter (704 channels). In the RCS experiment a calorimeter was operated for the first time at an effective electron-nucleon luminosity of $10^{39} \mathrm{~cm}^{2} / \mathrm{s}$ [9] (a $40 \mu \mathrm{A}$ electron beam on a $6 \% \mathrm{Cu}$ radiator followed by a $15 \mathrm{~cm}$ long liquid hydrogen target). In the test run and later in other kinematics of the RCS experiment we observed that the counting rate of the calorimeter, $f$, had an exponential dependence on the threshold, $E_{t h r}$, which could be presented as:

$f=A \times \exp \left(-B \times E_{t h r} / E_{\max }\right)$,

where $E_{\max }$ is the maximum energy of an elastically scattered electron for a given scattering angle, $A$ an angledependent constant, and $B$ a universal constant $\approx 9 \pm 1$. The angular variation of the constant $A$, after normalization to a fixed luminosity and the calorimeter solid angle, is less than a factor of 2 for the RCS kinematics. Figure 3 shows the counting rate for the conditions of the test run. The observed fast fall of the rate presents an important opportunity to have a relatively low trigger rate in the calorimeter, even at a high luminosity. However, on-line use of the calorimeter signal requires a set of summing electronics and careful equalizing and monitoring of the channels calibration during the experiment.

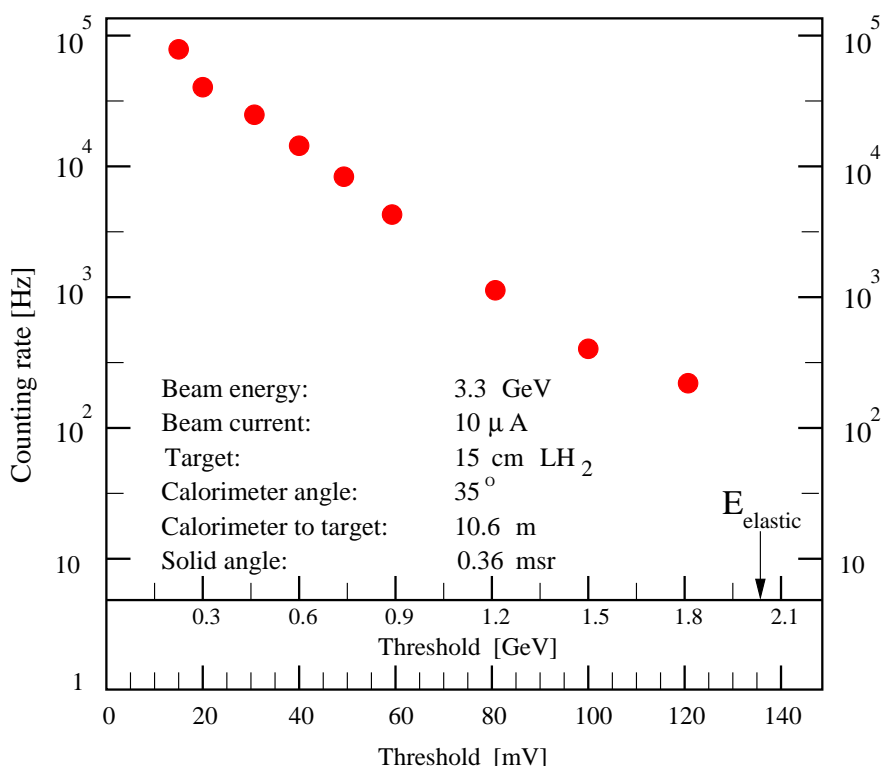

Fig. 3. Calorimeter trigger rate vs threshold level.

\section{Calorimeter}

The concept and technology of a fine-granularity electromagnetic lead-glass calorimeter (GAMS) was developed by Yu. Prokoshkin and collaborators at Serpukhov (Russia) [10]. Nowadays, the GAMS type concept has been applied in a large number of high-energy experiments at JLab, Serpukhov, CERN, FNAL and DESY for the registration of high-energy electrons and photons; see e.g. [11]. The 


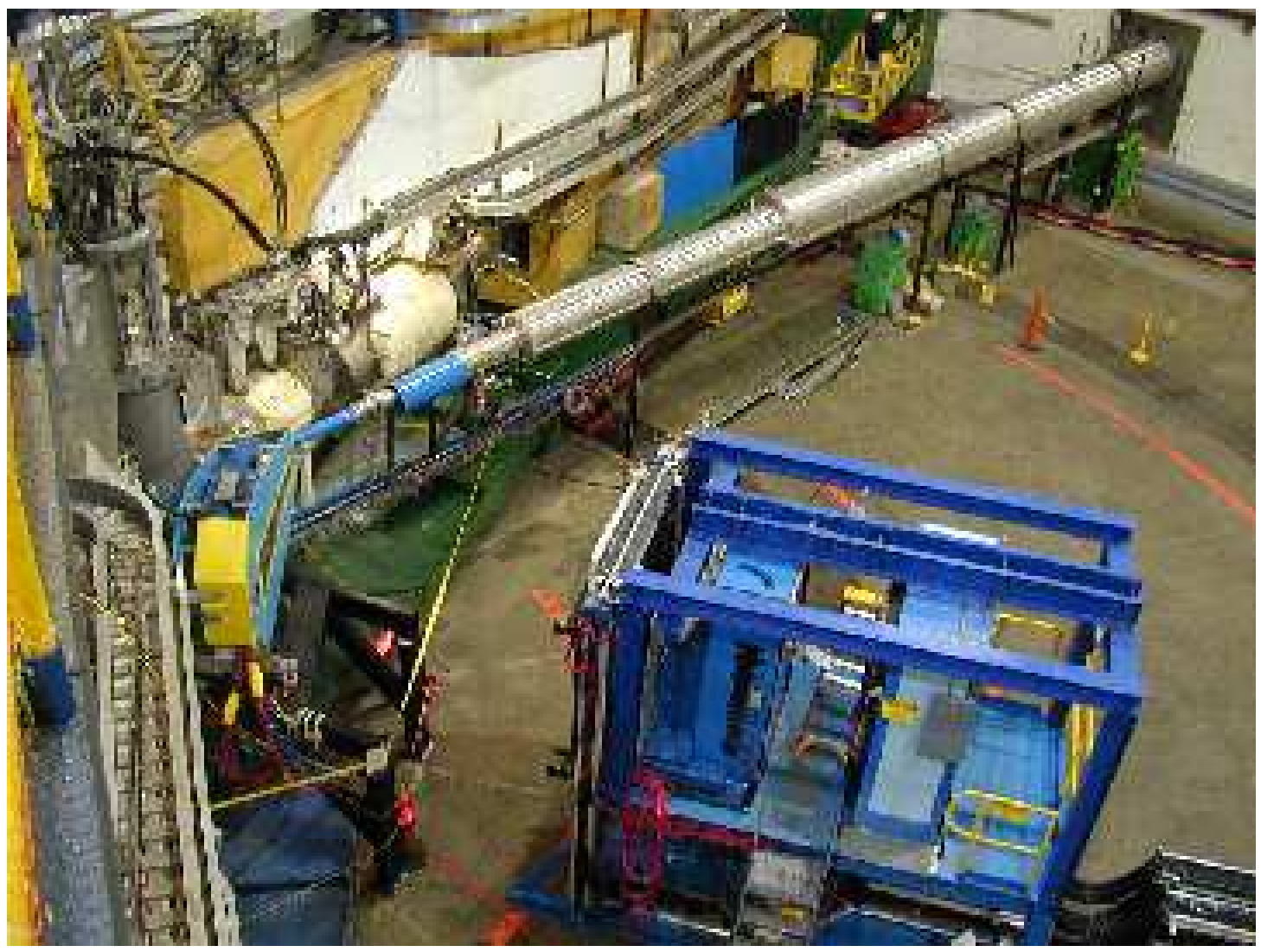

Fig. 2. A picture of the set-up for experiment E99-114.

present RCS calorimeter has many features which are similar to the Serpukhov design. The RCS calorimeter set-up is shown in Fig. 4. The main components are:

- the lead-glass blocks;

- a light-tight box containing the PMTs;

- a gain-monitoring system;

- two segmented veto hodoscopes;

- the front-end electronics;

- an elevated platform;

- a lifting frame.

The calorimeter frame hosts a matrix of $22 \times 32$ lead-glass blocks with associated PMTs and HV dividers. In front of the lead-glass blocks there is a sheet of UVT lucite, which was used to distribute a calibration light pulse among all 704 blocks. The light-tight box provides protection of the PMTs from ambient light and contains an air-cooling system and the HV and signal cable systems. Two veto hodoscopes, operating as Čerenkov counters with UVT lucite as a radiator, are located in front of the calorimeter. The front-end electronics located a few feet from the detector were assembled in three relay racks. They are comprised of 38 analog summers, trigger logic and patch panels. The elevated platform was needed to bring the calorimeter to the level of the beam line. The lifting frame was used to reposition the calorimeter by means of an overhead crane. Such an operation, which takes about 2 hours, was done more than 25 times during the E99-114 experiment.

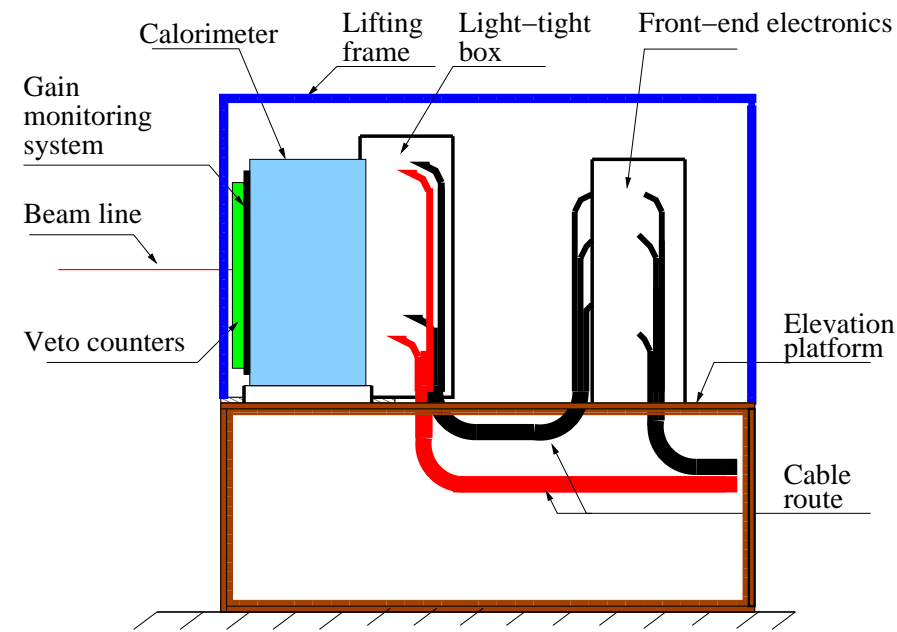

Fig. 4. Schematic side view of the calorimeter set-up.

\subsection{Calorimeter Design}

The main frame of the calorimeter is made of 10 " wide steel C-channels. Inside the frame a thick flat $\mathrm{Al}$ plate was bolted to the bottom. The second Al plate was installed vertically and aligned to $90^{\circ}$ with respect to the first one by means of alignment screws (see Fig. 5). Another set of screws, mounted in the main frame on the opposite side to the vertical alignment plate and on the top, was used to 
compress all gaps between the lead-glass modules and to fix their positions. The load was applied to the lead-glass through 1 " $\times 1$ " $\times 0.5$ " plastic plates and a $0.125 "$ rubber pad. There are a 1 " wide stainless steel strips of 0.004 " thickness between every two columns of lead-glass modules. These strips, stretched between the top and the bottom of the main frame, assisted the alignment of the blocks' geometry.

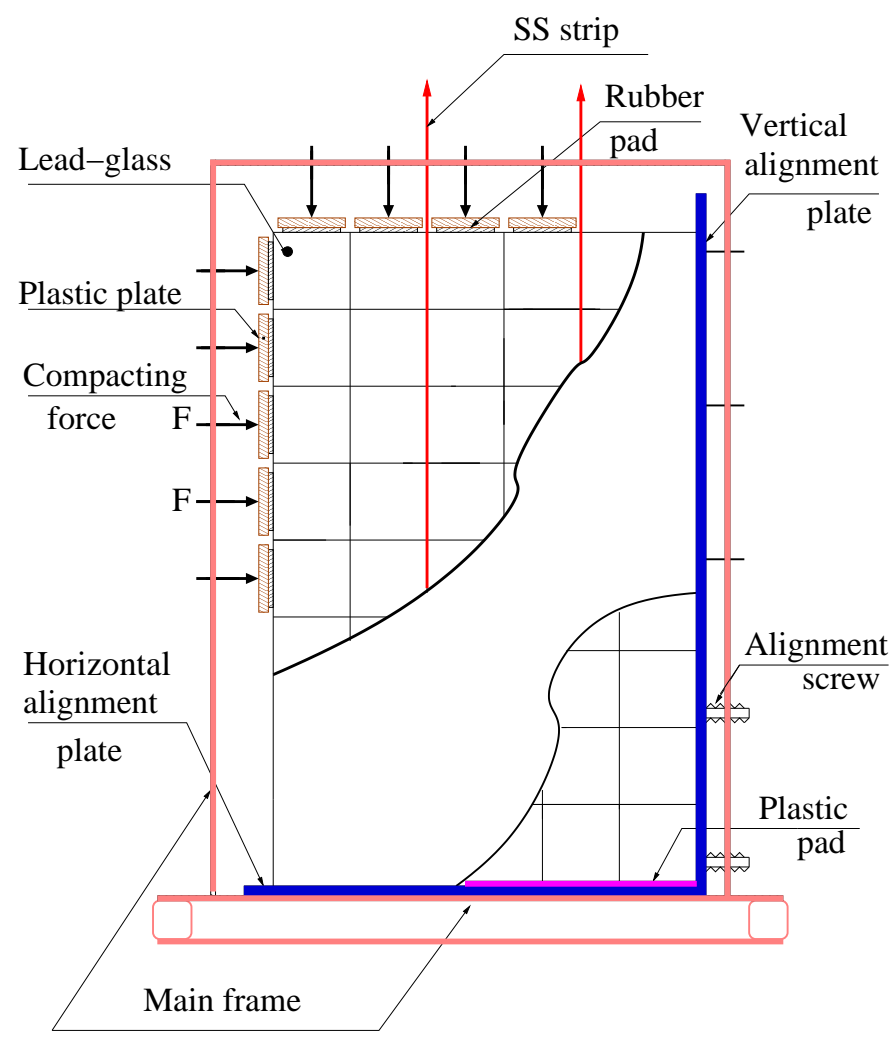

Fig. 5. Sectional front view of the calorimeter.

\subsubsection{Air cooling}

All PMTs and HV dividers are located inside a light-tight box (see Fig. 6). The current on each divider is $1 \mathrm{~mA}$; therefore, simultaneous operation of all PMTs would cause the temperature inside the box to rise by $50-70^{\circ} \mathrm{C}$. An air-cooling system was developed to prevent the PMTs from overheating, and to achieve a stable operation of the calorimeter. The air supply was provided by two parallel oil-less regenerative blowers R4110-2 type. The blowers supply air at a maximum pressure of 52" water and a maximum flow of 92 CFM. The air is distributed via the vertical collector tubes and a large number of outlets directing air towards to the HV dividers. Temperature sensors were installed in several positions inside the box. When the temperature value on one of the sensors exceeds a preset limit, the HV on the PMTs is turned off by an interlock system. The air line is equipped with a flow switch FST-321-SPDT included in the interlock system. The average temperature

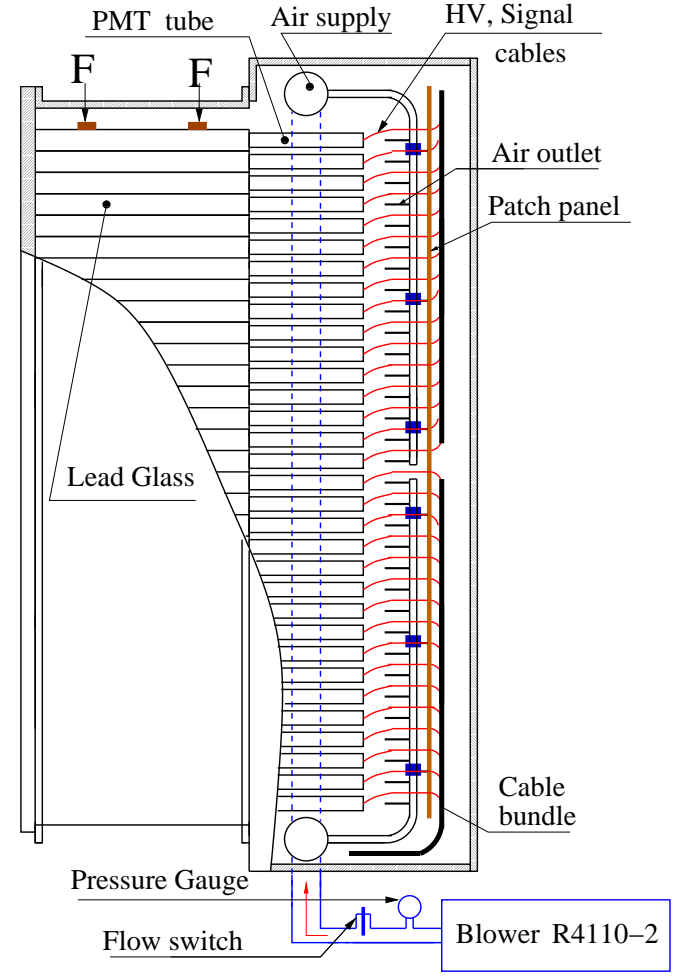

Fig. 6. A scheme of the calorimeter air cooling and cables.

inside the box was not more than $55^{\circ} \mathrm{C}$ during the production run.

\subsubsection{Cabling system}

Simple and reliable cabling is one of the important items in multichannel detectors. An important feature of the cabling system is easy access to the PMTs and the HV dividers for installation and repair. The system includes:

- 1' long HV and signal pig-tails soldered to the HV divider;

- patch panels with the lemo and the HV connectors;

- 10' long cables from those patch panels to the front-end electronics and the HV distribution boxes;

- HV distribution boxes;

- BNC-BNC patch panels for the outputs of the front-end modules;

- BNC-BNC patch panels on the DAQ side for the analog signals;

- BNC-lemo patch panels on the DAQ side for the vetocounter lines.

Figure 7 shows the cabling arrangement inside the box. The patch panels in the box are custom-made and mounted on the air supply tubes. They have the ability to swing to the side to allow access to the PMTs and the HV dividers. The box has two moving doors which interlock to the HV supply. In order to reduce the diameter of the cable bundle from the PMT box, RG-174 (diameter 0.1") cable was used for the PMT signals, and a twisted pair for the HV connection (two individually insulated inner 26 AWG conductors with an overall flame-retardant PVC jacket, part number 00121803 from the General Wire Product company). The box- 


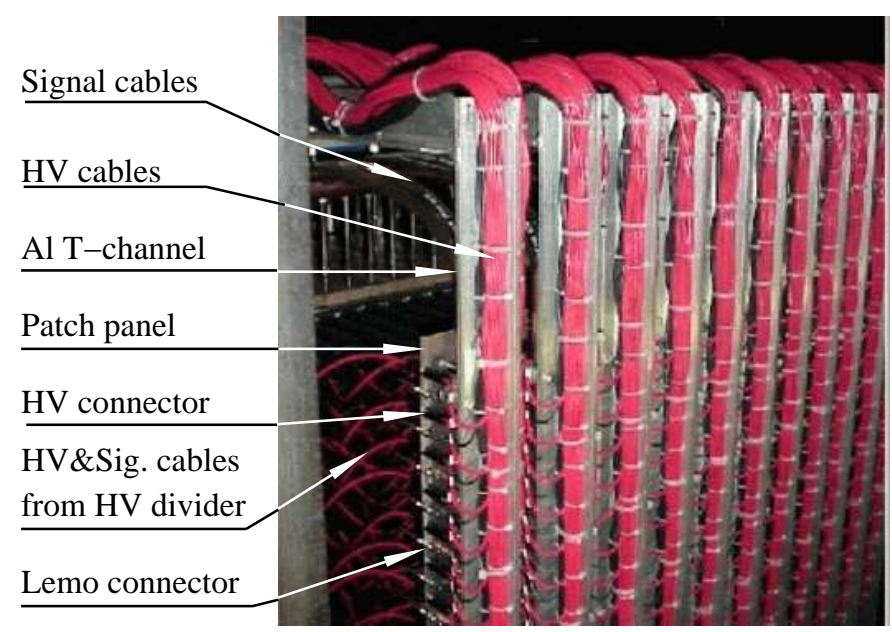

Fig. 7. A picture showing the cabling inside the PMT box.

patch panels used for the HV lines each convert 24 of the above twisted pairs (single HV line) to the multi-wire HV cables (the part 001-21798 made by General Wire Product), which run to the HV power supply units located in the shielded area near DAQ.

\subsection{Lead-Glass Counter}

The basic components of the segmented calorimeter are the TF-1 lead-glass blocks and the FEU 84-3 photo-tubes. In the 1980s the Yerevan Physics Institute (YerPhI) purchased TF-1 lead-glass blocks of $4 \mathrm{~cm} \times 4 \mathrm{~cm} \times 40 \mathrm{~cm}$ size and FEU 84-3 photo-tubes of $34 \mathrm{~mm}$ diameter (the active photo-cathode diameter is $25 \mathrm{~mm}$ ) for the construction of a calorimeter to be used in several experiments at the YerPhI synchrotron. In January of 1998 the RCS experiment at CEBAF was approved and soon after per an MOU between JLab and YerPhI the components of the calorimeter were sent to JLab. All PMTs had to pass a performance test with the following selection criteria: a dark current less than $30 \mathrm{nA}$, a gain of $10^{6}$ with stable operation over an extended period of time, a linear dependence of the PMT response on the incident light intensity. We selected 704 PMTs out of the 900 available. The mechanical properties and dimensional tolerances were checked for all lead-glass blocks: a length of $400 \pm 2 \mathrm{~mm}$ and transverse dimensions of $40 \pm 0.1 \mathrm{~mm}$.

\subsubsection{Design of the counter}

In designing the individual counters for the RCS calorimeter, much attention has been paid to reliability, simplicity and the possibility to quickly replace a PMT and/or voltage divider. The individual counter design is shown in Figure 8 . The titanium flange has been glued to one of the leadglass block ends by means of EPOXY-190. Titanium was selected because its thermal expansion coefficient is very close to that of the lead glass. The PMT housing was bolted to the Ti flange. The housing was made of an anodized $\mathrm{Al}$ flange and an $\mathrm{Al}$ tube. Inside the housing there are the PMT with a $\mu$-metal shield, the HV divider, a spring, and a spring holder. The optical contact between the PMT and the lead-glass block is achieved by means of optical grease, type BC-630 (Bicron), which increased the amount of light detected by the PMT by $30-40 \%$. The PMT was pressed to the lead-glass block by means of a spring, which pushed the $\mathrm{HV}$ base with a force of $1 \mathrm{lb}$. Such a large force is essential for the stability of the optical contact over time at the elevated temperature of the PMTs. The glue-joint between the lead glass and the Ti flange, which holds that force, failed after several months in a significant fraction (up to $5 \%$ ) of the counters. An alternative scheme of compression was realized in which the force was applied to the PMT housing from the external bars placed between the PMT and the patch-panel assembly. Each individual lead-glass block was

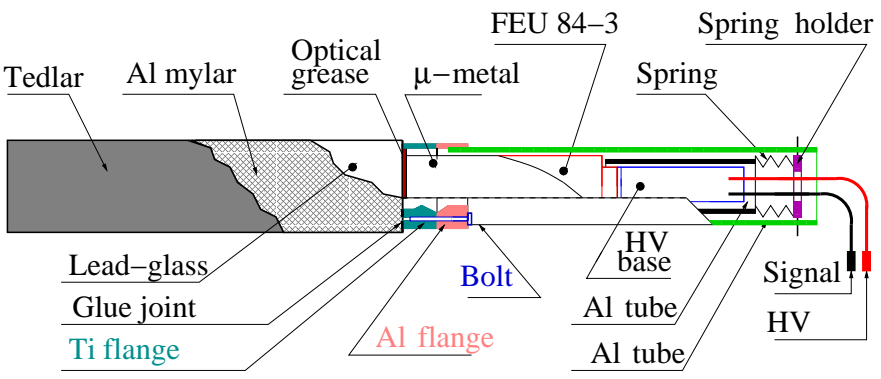

Fig. 8. Structure of the lead-glass module.

wrapped in aluminized mylar film and black tedlar for optimal light collection and inter-block isolation. Single-side aluminized mylar film was used with the Al layer on the side opposite the glass. Such an orientation of the film limits the diffusion of $\mathrm{Al}$ atoms into the glass and the non-oxidized surface of aluminum, which is protected by mylar, provides a better reflectivity. The wrapping covers the side surface of the lead-glass block, leaving the front face open for the gain monitoring. The signal and the HV cables are each one foot long. They are soldered to the HV divider on one end and terminated with Lemo@00 and D01EPB306XST connectors on the other end (Hypertronix).

\subsection{2. $H V$ divider}

At the full luminosity of the RCS experiment $\left(10^{39} \mathrm{~cm}^{2} / \mathrm{s}\right)$ the background energy load per lead-glass block reaches a level of $10^{8} \mathrm{MeV}$ per second. The electronic scheme and the selected scale of $1 \mathrm{MeV}$ per ADC channel ( $50 \mathrm{fC}$ ) resulted in an average anode current of $5 \mu \mathrm{A}$ due to background load. A high-current HV base ( $1 \mathrm{~mA}$ ) has been chosen to reduce the effect of the beam intensity variation on PMT amplitude and energy resolution to the level of $1 \%$. The scheme of the $\mathrm{HV}$ base is shown in Fig. 9. The values of the resistors are $R(1-10)=100 k \Omega, R 11=130 k \Omega, R 12=150 k \Omega, R 13=$ $200 k \Omega, R 14=150 k \Omega, R 15=10 k \Omega, R 16=10 M \Omega, R 17=$ $4 k \Omega$. The capacitance $\mathrm{C}$ is $10 \mathrm{nF}$. The base is a C-type with an intermediate progressive type voltage distribu- 
tion. According to the specification data for the FEU 84-3 the maximum operation voltage is $1900 \mathrm{~V}$. Therefore we selected a nominal voltage value of $1800 \mathrm{~V}$ and a current value in the voltage divider of $1 \mathrm{~mA}$.

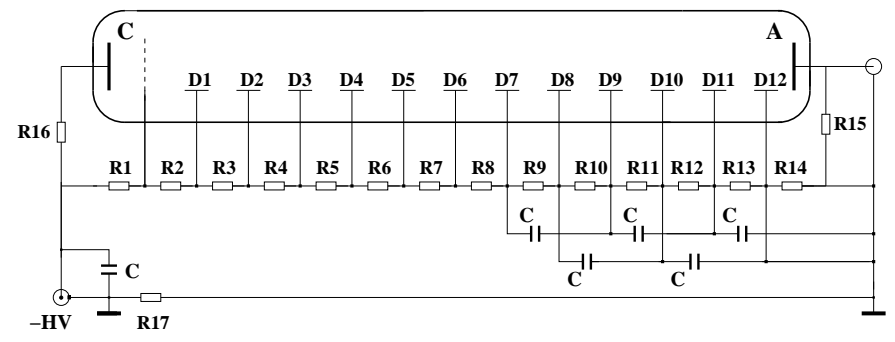

Fig. 9. Schematic of the high-voltage divider for the FEU 84-3 PMT.

\subsection{Electronics}

The calorimeter electronics were distributed over two locations; see the block diagram in Fig. 10. The first group of modules (front-end) is located in three racks mounted on the calorimeter platform in close vicinity to the lead-glass blocks. These are the trigger electronics modules assembled from custom-made and commercially available NIM units:

- 38 custom-made analog summing modules used for levelone signal summing 4 ;

- 14 linear fan-in/fan-out modules, LeCroy model 428F type, for a second-level signal summation;

- four Phillips Scientific model 706 discriminators;

- a master OR circuit, realized with Phillips Scientific logic units: four model 755 and one model 757 modules;

- several additional NIM modules used to provide auxiliary trigger signals for the calorimeter calibration with cosmics and for the PMT gain-monitoring system.

The second group of electronic modules is placed behind a radiation-protecting concrete wall and includes charge and time digitizers as well as equipment of the Data Acquisition System and of the High Voltage supply and slow-control systems. All 704 lead-glass PMT signals and 56 SUM-32 signals are digitized by LeCroy 1881M FastBus ADC modules. In addition, 56 SUM-32 discriminator pulses are directed to scalers and to LeCroy 1877 FastBus TDCs. More information about the electronics is presented in Section 5 . The signals between these locations are conducted via patch-panels and coaxial cables; the total amount is 1040 signal and $920 \mathrm{HV}$ lines. The length of the signal cables was about $100 \mathrm{~m}$; they serve also as delay lines allowing the timing of the signals at the ADC inputs to be properly set with respect to the ADC gate, formed by the experiment trigger. The cables are placed on a chain of bogies, which permits the calorimeter platform to be moved in the experimental hall without disconnecting the cables. This is needed for a quick change of kinematics, which is very important for experiments requiring measurements

\footnotetext{
4 This module was designed by S. Sherman, Rutgers University.
}

at many calorimeter positions (25 in the case of the RCS experiment).

\subsubsection{Trigger scheme}

The fast on-line photon trigger is based on PMT signals from the calorimeter counters. The principle of its operation is a simple constant-threshold method - a logic pulse is produced if the energy deposition in the calorimeter is above a given magnitude. Since the Molière radius of the calorimeter material is $R_{M} \approx 3.0 \mathrm{~cm}$, the transverse size of the electromagnetic shower in the calorimeter exceeds the size of a single lead-glass block. This enables a good position sensitivity of the device. At the same time this makes it mandatory for the trigger scheme to sum up signals from several adjacent counters to get a signal proportional to the energy deposited in the calorimeter.

A simplest (from a trigger electronics point of view) realization of the trigger would be a summation of all blocks and directing a resulting signal to a single discriminator. However, such a design is inappropriate for a high-luminosity experiment due to the very high background level. The other extreme approach would be to form a summing signal for a minimal group, which includes a counter, hit by an initial photon and 8 adjacent counters, thus forming a $3 \times 3$ counters structure. This should be done for every lead-glass block, except for those at the calorimeter's edges. Such a scheme provides the optimal signal-to-background ratio, but it looks impractical because it would require 600 channels of an analog splitter $\rightarrow$ analog summer $\rightarrow$ discriminator circuit followed by a 600 -inputs fan-in module. The trigger scheme that we adopted is a trade-off between the above extreme cases - see the principle of its logic in Fig. 11. The
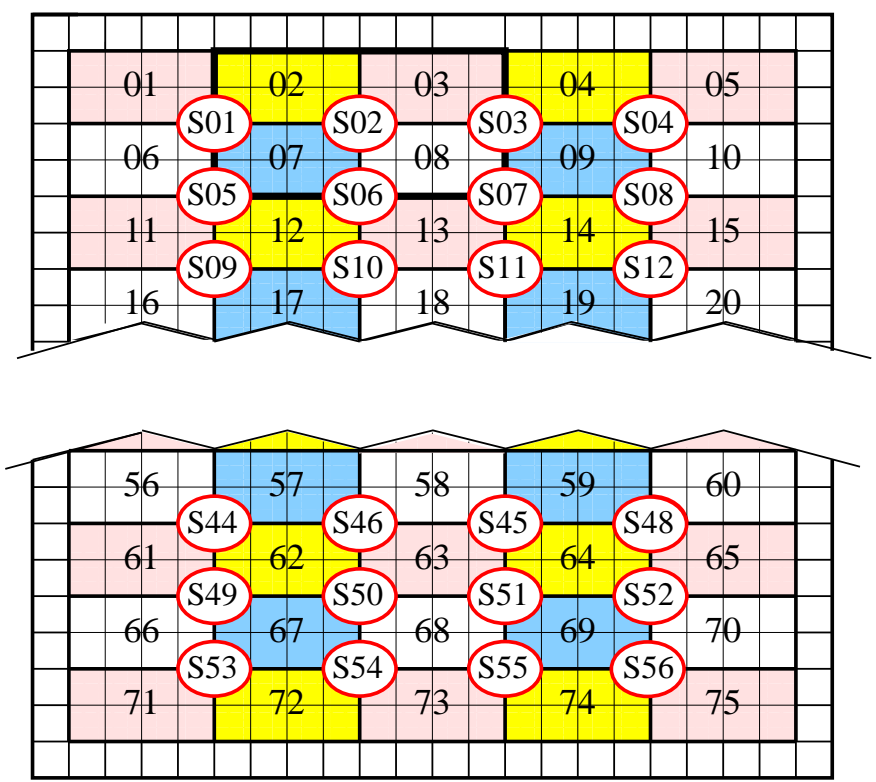

Fig. 11. The principle of two-level summation of signals for the hardware trigger: 75 eight-block sub-arrays and 56 overlapping groups of four sub-arrays forming $S U M$-32 signals labeled as S01-S56. In outlined example the sums 02,03,07, and 08 are forming a S02 signal. 
Calorimeter Platform

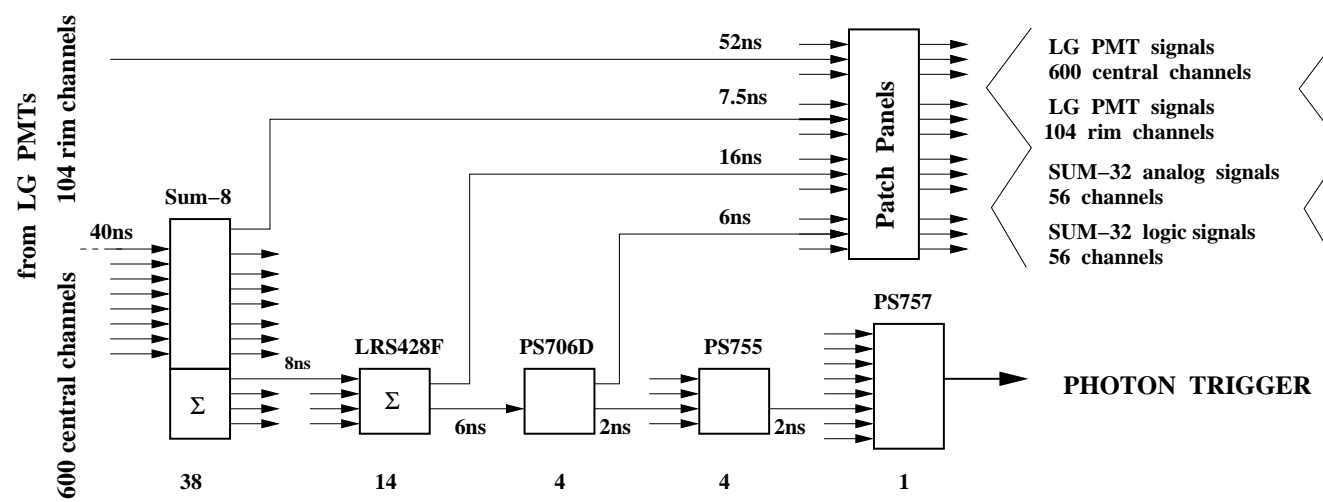

Fig. 10. A block diagram of the calorimeter electronics.
DAQ Platform

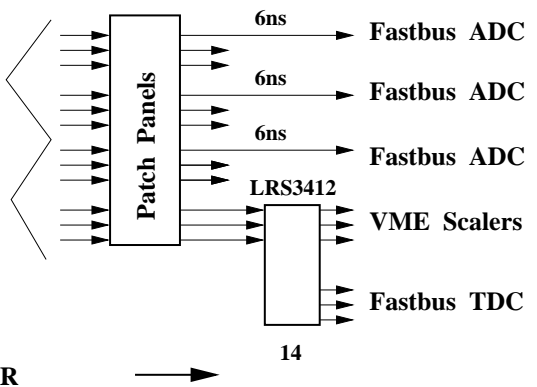

To Trigger Superviser scheme contains two levels of analog summation followed by the discriminators and the OR-circuit. It has realized the following functions:

- the signals from each PMT in the $752 \times 4$ sub-arrays of adjacent lead-glass blocks, excluding the outer-most blocks, are summed in a custom-made analog summing module to give a $S U M-8$ signal;

- these signals, in turn, are further summed in overlapping groups of four in LeCroy LRS428F NIM modules to produce $56 S U M$-32 signals. Thus, each $S U M$-32 signal is proportional to the energy deposition in a calorimeter segment of 4 blocks high and 8 blocks wide, i.e. $16 \times 32$ $\mathrm{cm}^{2}$; This amounts to only $5 \%$ of the calorimeter acceptance; however, for any photon hitting the calorimeter (except for those which hit the edges) there will be at least one segment which contains the whole electromagnetic shower.

- the SUM-32 signals are sent to constant-threshold discriminators, from which the logical pulses are ORed to form the photon singles trigger T1; see Section 5. The discriminator threshold is remotely adjustable. The threshold was typically set around half of the RCS photon energy at a given kinematic point.

\subsection{Gain monitoring system}

The detector is equipped with a system, that distributes light pulses to each calorimeter module. The purpose of this system is to provide a quick way to check the detector operation, to calibrate the dependence of the signal amplitudes on the HV applied, and to monitor the gains of all detector channels during the experiment. The detector response to photons of a given energy may drift with time, due to drifts of the photo-multiplier gains, and due to changes in the glass transparency, caused by radiation damage. In designing the gain-monitoring system we used ideas developed for a large lead-glass calorimeter at BNL[15].

The system includes a stable light source and a system to distribute the light to all calorimeter modules. The light source consists of an LN300 nitrogen laser 5 and a wavelength shifter. The LN300 laser provides $5 \mathrm{~ns}$ long, $300 \mu \mathrm{J}$ ultraviolet light pulses of $337 \mathrm{~nm}$ wavelength. The light passes through an absorber, including a remotely controlled wheel with several calibrated absorbers, lenses, and arrives at a wavelength shifter. The wavelength shifter used is a 1" diameter semi-spherical piece of plastic scintillator, in which the ultraviolet light is fully absorbed and converted to a blue $(\sim 425 \mathrm{~nm})$ light pulse, radiated isotropically. Around the scintillator semi-sphere about 40 plastic fibers $2 \mathrm{~mm}$ thick and $4 \mathrm{~m}$ long are arranged, transporting the light to the detector. A lucite plate is mounted adjacent to the front face of the lead-glass calorimeter, covering the full aperture of the calorimeter (see Fig.12). The front faces of the lead-glass modules are open to light. The fibers transport the light from the laser to the side of the lucite plate. The light passes through the length of the plate, and due to light scattering in the lucite the plate slightly glows. This glowing is seen by the lead-glass calorimeter modules. Such a system provides a rather uniform light collection for all modules.

We checked possible cross-talk between adjacent modules, caused by light leaving the front face of a module and being reflected by the lucite plate to the face of another module. Light was sent through a fiber to the rear side of a module and the signal in the adjacent module was measured. Such a test does not simulate properly the light coming from a shower originated inside the module and can be used only for general guidance. The results depend on the distance between the modules and the plate and are different for different modules, depending on the shape of their edges. A cross-talk of $\sim 1 \%$ was observed. In order to eliminate this cross-talk a mask was inserted between the lucite plate and the detector face. The mask is built of $12.7 \mathrm{~mm}$ thick black plastic and contains a $2 \mathrm{~cm} \times 2 \mathrm{~cm}$ hole in front of each module. The mask reduces the cross-talk by at least a factor of 100 .

The light pulse coming out of the laser was attenuated, typically by two orders of magnitude, and monitored using

\footnotetext{
5 Manufactured by Laser Photonics, Inc, FL 32826, USA.
} 


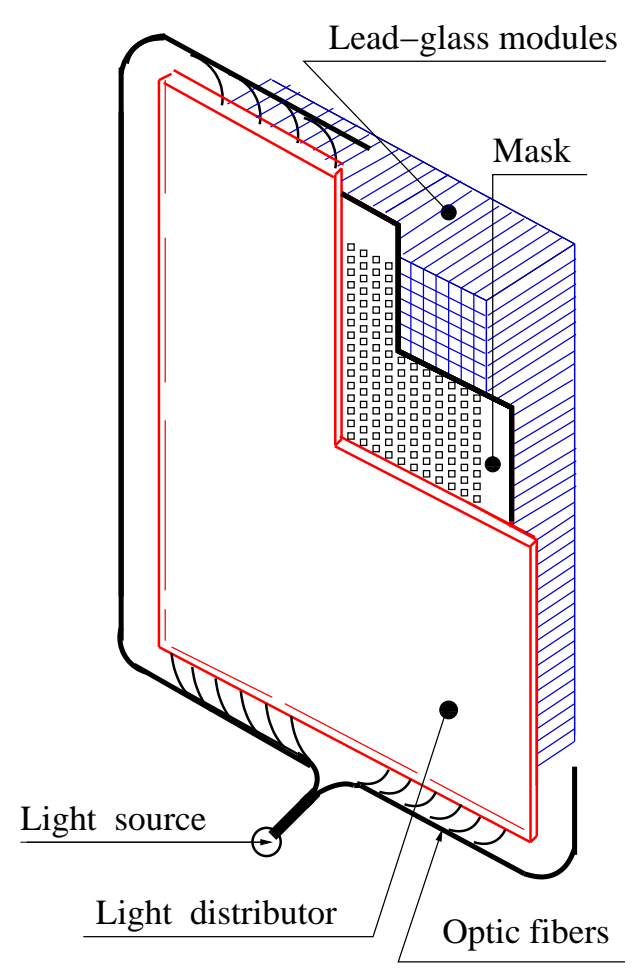

Fig. 12. Gain-monitoring system.

a silicon photo-diode $\mathrm{S} 1226-18 \mathrm{BQ}{ }^{6}$ mounted at $150^{\circ}$ to the laser beam. The beam passed through a thin tilted quartz plate positioned at $15^{\circ}$ to the beam, which reflects a small fraction of light to the detector. The optical system at the exit of the laser, including the photo-diode and the scintillator, is thermally stabilized. The amplitude variance of the signals from the laser is about $0.7 \%$ on a pulse-topulse basis. The signal from the scintillator is monitored using two photo-multiplier tubes. The light is delivered to the tubes by fibers and attenuated by optical filters. During operation at a repetition rate of $1 \mathrm{~Hz}$ the laser yield drops by about $0.1 \%$ per day, while the scintillator yield drops by about $2 \%$ per day. The signals from the photo-diode and two monitoring photo-multipliers are used to normalize the light yield.

Two groups of 20 fibers delivered the light to the bottom and to the top sides of the plate, with air gaps of about $2 \mathrm{~mm}$ between the fiber edge and the plate. The detector response was uniform with an RMS of about $10 \%$ at the central part of the detector (about $70 \%$ of all modules), while for 3 top and 3 bottom rows the responses varied by a factor as large as 2 .

The system described was useful for detector testing and tuning, as well as for troubleshooting during the RCS experiment. Gain monitoring during the RCS experiment was found not to be practical. The reason is that the main radiation damage occurred at the faces of the modules within a depth of about $2-4 \mathrm{~cm}$. The monitoring light passes through the damaged area, while an electromagnetic shower has its maximum at a depth of about $10 \mathrm{~cm}$. Therefore the moni-

\footnotetext{
6 Manufactured by Hamamatsu Photonics, Hamamatsu, Japan.
}

toring signals drop relatively stronger than the signals from particles.

\section{Veto Hodoscopes}

To insure identification of the photon scattered in the complicated environment of the mixed electron-photon beam we developed veto hodoscopes using UVT lucite as a Cerenkov radiator. The veto hodoscopes are located in front of the calorimeter. The first hodoscope has 80 counters oriented vertically and the second 110 counters oriented horizontally; see Fig. 13.

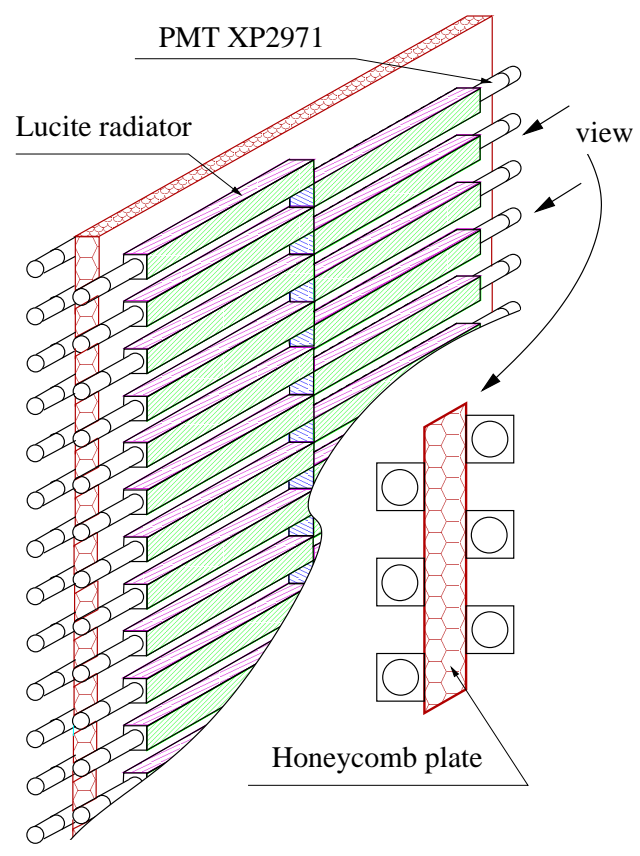

Fig. 13. Cut-off view of the "horizontal" veto hodoscope.

Each counter is made of a UVT lucite bar with a PMT directly glued onto it. Figure 14 shows the concept of the veto counter. The lucite bar of $2 \times 2 \mathrm{~cm}^{2}$ cross section was glued to a XP2971 PMT and wrapped in aluminized mylar and black tedlar. Counters were mounted on a light honeycomb

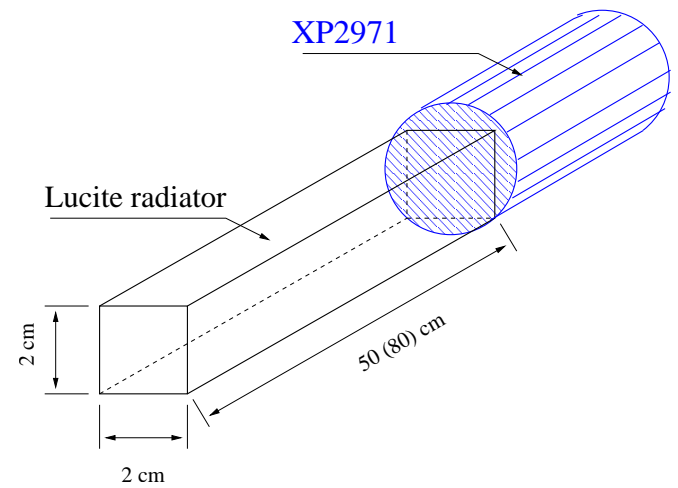

Fig. 14. Schematic of the veto counter. 
plate via an alignment groove and fixed by sticky tape. The counters are staggered to allow for the size of the PMTs and the counters to overlap.

The average PMT pulse generated by a high-energy electron corresponds to 20 photo-electrons. An amplifier, powered by the HV line current, was added to the standard high-voltage divider, so that the PMT gain could be reduced by a factor of 10 [12]. After gain matching by cosmic rays a good rate uniformity was achieved; see the rate distribution of the counters during the RCS experiment in Fig. 15, where the regular variation reflects the shielding effect due to the staggered arrangement of the counters. A significant reduction of the rate (by a factor of 5) was

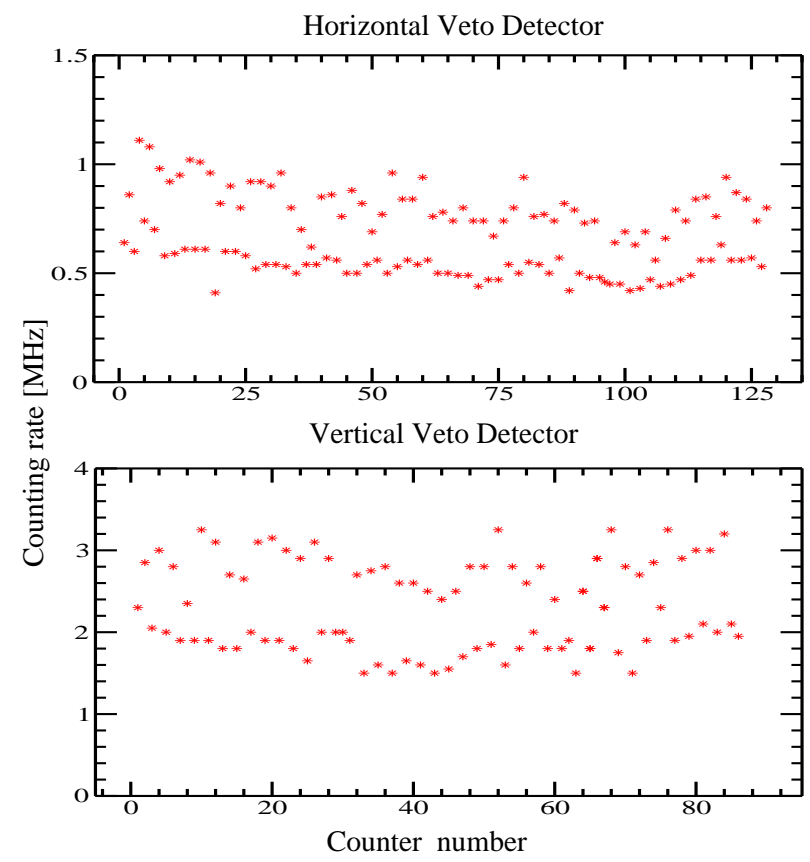

Fig. 15. The counting rate in the veto counters observed at luminosity of $1.5 \cdot 10^{38} \mathrm{~cm}^{-2} / \mathrm{s}$.

achieved by adding a 2" polyethylene plate in front of the hodoscopes. The veto plane efficiency measured for different beam intensities is shown in Table 1. It drops significantly at high rate due to electronic dead-time, which limited the beam intensity to $3 \mu \mathrm{A}$ in the production configuration.

An analysis of the experimental data showed that the deflection of the electrons by the magnet provided a sufficiently clean photon event sample, so the veto hodoscopes were not used during most of data taking runs.

\section{High Voltage System}

Each PMT high-voltage supply was individually monitored and controlled by the High Voltage System (HVS). The HVS consists of six power supply crates of LeCroy type 1458 with high-voltage modules type $1461 \mathrm{~N}$, a cable system, and a set of software programs. The latter allows to control,
Table 1

The efficiency of the veto hodoscopes and the rate of a single counter at different beam currents. The detector was installed at $30^{\circ}$ with respect to the beam at a distance $13 \mathrm{~m}$ from the target. The radiator had been removed from the beam path, the deflection magnet was OFF and the 2" thick polyethylene protection plate was installed.

\begin{tabular}{|c|c|c|c|c|}
\hline Run & $\begin{array}{c}\text { Beam } \\
\text { current } \\
{[\mu \mathrm{A}]}\end{array}$ & $\begin{array}{c}\text { Rate of the } \\
\text { counter V12 } \\
{[\mathrm{MHz}]}\end{array}$ & $\begin{array}{c}\text { Efficiency } \\
\text { horizonal } \\
\text { hodoscope }\end{array}$ & $\begin{array}{c}\text { Efficiency } \\
\text { vertical } \\
\text { hodoscope }\end{array}$ \\
\hline 1811 & 2.5 & 0.5 & $96.5 \%$ & $96.8 \%$ \\
\hline 1813 & 5.0 & 1.0 & $95.9 \%$ & $95.0 \%$ \\
\hline 1814 & 7.5 & 1.5 & $95.0 \%$ & $94.0 \%$ \\
\hline 1815 & 10. & 1.9 & $94.4 \%$ & $93.0 \%$ \\
\hline 1816 & 14. & 2.5 & $93.4 \%$ & $91.0 \%$ \\
\hline 1817 & 19 & 3.2 & $92.2 \%$ & $89.3 \%$ \\
\hline
\end{tabular}

monitor, download and save the high-voltage settings and is described below in more detail. Automatic HV monitoring provides an alarm feature with a verbal announcement and a flashing signal on the terminal. The controls were done over an Ethernet network using TCP/IP protocol. A Graphical User Interface running on Linux PC provides access to all features of the LeCroy system, loading the settings and saving them in a file. An sample distribution of the HV settings is shown in Fig. 16.

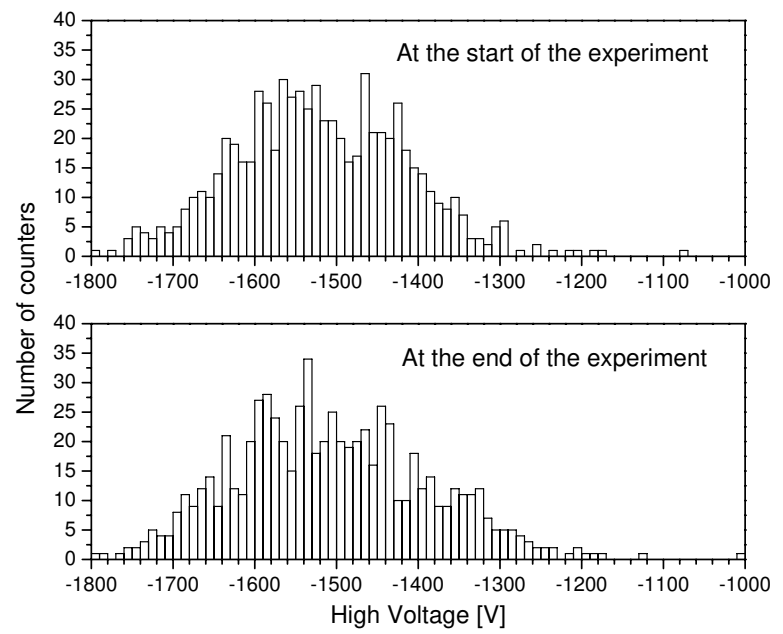

Fig. 16. The HV settings for the calorimeter PMTs.

The connections between the outputs of the high-voltage modules and the PMT dividers were arranged using $100 \mathrm{~m}$ long multi-wire cables. The transition from the individual HV supply outputs to a multi-wire cable and back to the individual PMT was arranged via the high-voltage distribution boxes, that are located inside the DAQ area, and the front-end patch panels outside the PMT box. These boxes have input connectors for individual channels on one side and two high-voltage multi-pin connectors (27 pins from FISCHER part number D107 A051-27) on the other side. 
High-voltage distribution boxes were mounted on the side of the calorimeter stand and on the electronics rack.

\section{Data Acquisition System}

Since the calorimeter was intended to be used in Hall A at JLab together with the standard Hall A detector devices, the Data Acquisition System of the calorimeter was part of the standard Hall A DAQ system. The latter uses CODA (CEBAF On-line Data Acquisition system) [13] developed by the JLab data-acquisition group. The calorimeter DAQ

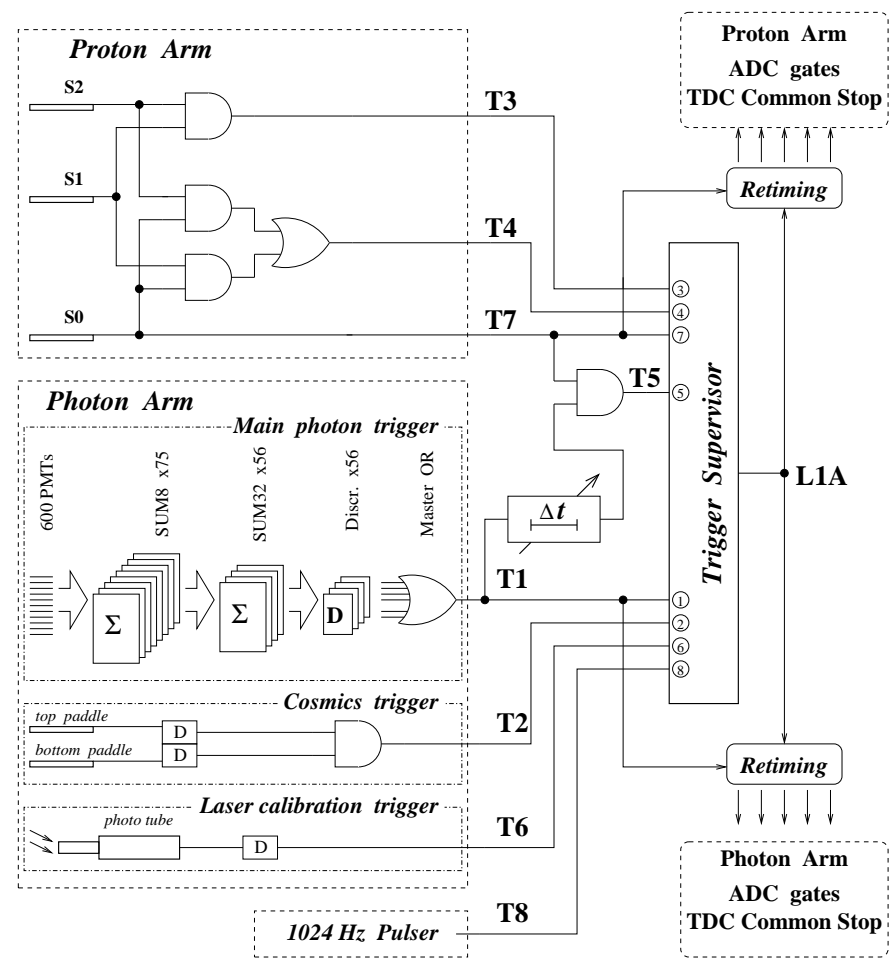

Fig. 17. Schematic diagram of the DAQ trigger logic.

included one Fastbus crate with a single-board VME computer installed using a VME-Fastbus interface and a trigger supervisor module [14], which synchronized the read-out of all information about given event. The most important software components were a Read-Out Controller (ROC) which runs on the VME computer under VxWorks OS and an Event Builder and an Event Recorder which run on a Linux workstation. For a detailed description of the Hall A DAQ system design and operation see [8] and references therein.

All 704 PMT signals and 56 SUM-32 signals were digitized by LeCroy 1881M FastBus ADC modules. The 56 SUM32 discriminator pulses were also directed to scalers and to LeCroy 1877 FastBus TDCs. During the RCS experiment the calorimeter was operating in conjunction with one of the High Resolution Spectrometers (HRS), which belong to the standard Hall A detector equipment [8]. The Hall A Data Acquisition System was able to accumulate data of several event types simultaneously. In the RCS experiment there were 8 types of trigger signals and corresponding event types. Trigger signals from HRS were generated by three scintillator planes: S0, S1 and S2 (see Fig. 8 in [8]). In the standard configuration the main single arm trigger in the spectrometer is formed by a coincidence of signals from $\mathrm{S} 1$ and $\mathrm{S} 2$. An alternative trigger, logically described as (S0 AND S1) OR (S0 AND S2), is used to measure the trigger efficiency. In the RCS experiment one more proton arm trigger was used, defined just by a hit in the $\mathbf{S 0}$ plane. Being the fastest signal produced in the proton arm, it was better suited to form a coincidence trigger with the photon calorimeter.

The logic of the Photon Arm single trigger is described in Section 2.3. A schematic diagram of the RCS experiment DAQ trigger logic is shown in Fig.17. Besides the main photon trigger there were two auxiliary triggers that serve to monitor the calorimeter blocks and electronics. The first was a photon arm cosmics trigger, which was defined by a coincidence between signals from two plastic scintillator paddles, placed on top and under the bottom of the calorimeter. The other trigger was the light-calibration (laser) trigger. The two-arm coincidence trigger was made from a time overlap of the main calorimeter trigger and the signal from the S0 scintillator plane in the HRS. The width of the spectrometer trigger pulse was set to $100 \mathrm{~ns}$, while the width of the photon trigger pulse was set to $10 \mathrm{~ns}$, and before sending to the coincidence circuit it was delayed in a programmable delay line. As a result, the coincidence events were synchronized with the photon trigger, and a correct timing relation between trigger signals from two arms was maintained for all 25 kinematic configurations of the RCS experiment. Finally, a $1024 \mathrm{~Hz}$ pulser generator signal formed a pulser trigger. It was used to measure the dead time of the electronics.

All 8 trigger signals were sent to the Trigger Supervisor module which starts the DAQ readout. Most inputs of the Trigger Supervisor can be individually pre-scaled. Triggers which were accepted by the DAQ were then re-timed with the scintillators of a corresponding arm to make gates for ADCs and TDCs. This re-timing removes trigger time jitter and makes the timing independent of the trigger type. Table 2 accumulates the information on the triggers and event types of the RCS experiment and shows typical prescale factors used during the data-taking.

\section{Calorimeter Performance}

The main characteristic of the calorimeter, its energy resolution, is shown in Table 3, as it was determined in different stages of the RCS experiment. The initial studies of the energy and position resolutions of the calorimeter were performed in two test runs [7] by using elastic electron scattering from the proton and the wire chamber in front of the calorimeter. We present the algorithms of energy cali- 
Table 2

A list of triggers used in the RCS experiment. Typical pre-scale factors which were set during the data-taking run (run \#1819) are shown.

\begin{tabular}{|c|c|c|}
\hline $\begin{array}{c}\text { Trigger } \\
\text { ID } \\
\end{array}$ & Trigger Description & $\begin{array}{c}\text { pre-scale } \\
\text { factor }\end{array}$ \\
\hline $\mathrm{T} 1$ & Photon arm singles trigger & 100,000 \\
\hline $\mathrm{T} 2$ & Photon arm cosmics trigger & 100,000 \\
\hline $\mathrm{T} 3$ & $\begin{array}{l}\text { Main Proton arm trigger: } \\
\text { (S1 } A N D \text { S2) }\end{array}$ & 1 \\
\hline $\mathrm{T} 4$ & $\begin{array}{l}\text { Additional Proton arm trigger: } \\
\text { (S0 } A N D \text { S1) OR (S0 AND S2) }\end{array}$ & 10 \\
\hline T5 & Coincidence trigger & 1 \\
\hline T6 & $\begin{array}{l}\text { Calorimeter light-calibration trig- } \\
\text { ger }\end{array}$ & 1 \\
\hline $\mathrm{T} 7$ & $\begin{array}{l}\text { Signal from the HRS S0 scintilla- } \\
\text { tor plane }\end{array}$ & 65,000 \\
\hline $\mathrm{T} 8$ & $1024 \mathrm{~Hz}$ pulser trigger & 1,024 \\
\hline
\end{tabular}

bration and position reconstruction used in the RCS data analysis. During the E99-114 production run elastic scattering data were taken frequently for the calorimeter energy calibration and for the determination of the calorimeter angular position. These data allow the evaluation of the degradation of the calorimeter resolution due to radiation damage.

Table 3

Calorimeter energy resolution at different stages of the RCS experiment.

\begin{tabular}{|l|c|c|c|c|c|}
\hline $\begin{array}{l}\text { Runs } \\
\text { used }\end{array}$ & $\begin{array}{c}\text { Average } \\
\text { energy } \\
{[\mathrm{GeV}]}\end{array}$ & $\begin{array}{c}\sigma_{E} / E, \\
\%\end{array}$ & $\begin{array}{c}\sigma_{E} / E \\
\text { at } \mathrm{E}_{\gamma}= \\
1 \mathrm{GeV}\end{array}$ & $\begin{array}{c}\text { Beam } \\
\text { current } \\
{[\mu \mathrm{A}]}\end{array}$ & $\begin{array}{c}\text { Accumu- } \\
\text { lated beam } \\
\text { charge }[\mathrm{C}]\end{array}$ \\
\hline 1488 & 1.32 & 4.9 & 5.5 & 5 & 0.5 \\
\hline $1811-12$ & 2.78 & 4.2 & 7.0 & 2.5 & 2.4 \\
$1811-18$ & 2.78 & 4.6 & 7.7 & $2.5-20$ & 2.5 \\
\hline 1930 & 3.39 & 4.2 & 7.7 & 40 & 4.4 \\
\hline 2125 & 2.83 & 4.9 & 8.2 & 25 & 6.6 \\
\hline 2593 & 1.32 & 9.9 & 11.3 & 38 & 14.9 \\
\hline 3365 & 1.31 & 8.4 & 9.6 & 41 & 29.2 \\
\hline
\end{tabular}

\subsection{Amplitude characteristics of the calorimeter}

At high luminosity, when reduction of the accidental and raw trigger rate is very important, the trigger threshold should be as close to the signal amplitude for elastic RCS photons as practical. However, there are several effects which generate jitter in the effective threshold level. The combination of a high threshold and large threshold jitter could lead to a loss of detection efficiency. The actual value of the threshold for an individual event has a significant uncertainty due to pile-up of the low-amplitude signals, fluctuations of the signal shape (mainly due to summing of the signals from the PMTs with different HV and transit time), and inequality of the gain in the individual counters. Figure 18 shows the spectra of signals in the largest cluster in the calorimeter. In data taking runs for kinematic 5C the threshold was set at $1.2 \mathrm{GeV}$. The event-to-event fluctuation of the effective threshold is $\pm 10 \%$. The width of the

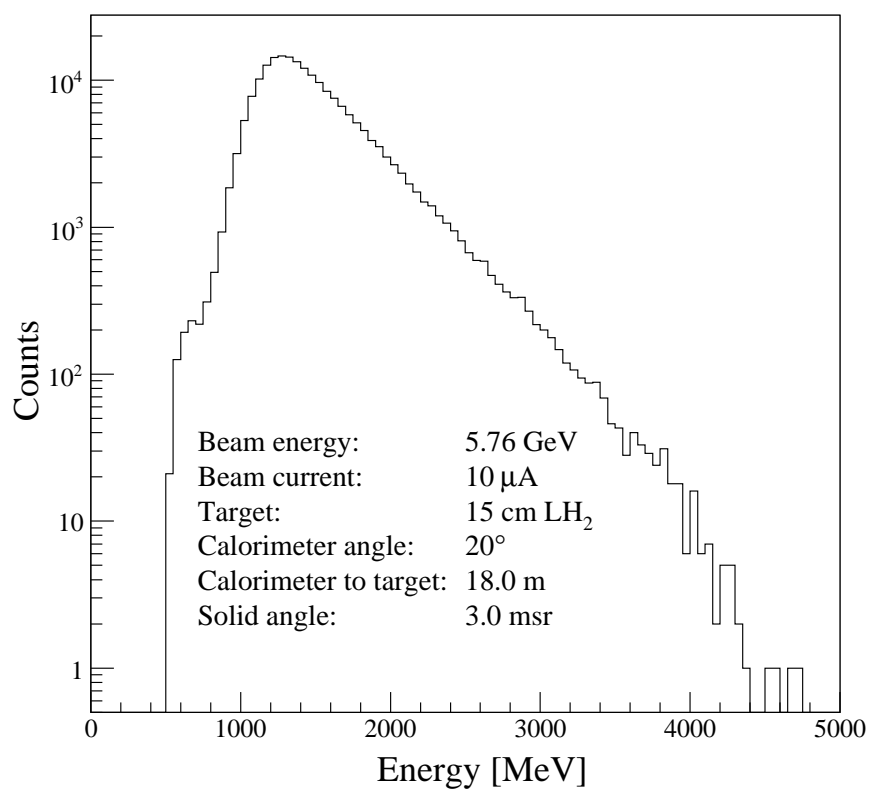

Fig. 18. Amplitude spectrum of the signals in the calorimeter for kinematic $5 \mathrm{C}$ with the radiator inserted into the beam path. The threshold at $1200 \mathrm{MeV}$ has a FWHM of $20 \%$.

ADC pedestals, shown in Fig. 19, allows to monitor and to estimate a contribution of the base line fluctuations to the energy resolution. For the case shown in Fig. 19 the width is about $6 \mathrm{MeV}$, which results in $20 \mathrm{MeV}$ contribution to the energy resolution for a 9 blocks cluster size.

Equalization of the counter gains was initially done with cosmic muons, which produce $20 \mathrm{MeV}$ energy equivalent light output per $4 \mathrm{~cm}$ path (muon trajectories perpendicular to the long axis of the lead-glass blocks). The calibration was done by using selected cosmic events for which the signal are large in both counters above and below the given counter. The final adjustment of each counter's gain was done by using calibration with e-p elastic events. This calibration provided PMT gain values which in average were different from the initial set by $20 \%$.

\subsection{Shower information analysis}

The event analysis realizes a search for a cluster (see a labeling scheme in Fig. 20), which is defined as:

(i) a cluster is a group of adjacent blocks;

(ii) a cluster occupies $9(3 \times 3)$ blocks of the calorimeter; 


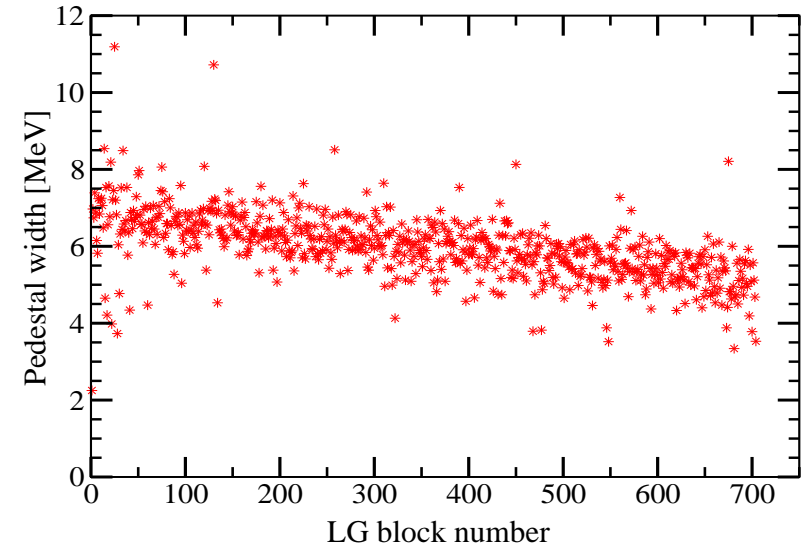

Fig. 19. The width of the ADC pedestals for the calorimeter in a typical run. The observed reduction of the width vs. the block number reflects the lower background at larger detector angle with respect to the beam direction.

(iii) the distribution of the shower energy deposition over the cluster blocks (so-called shower profile) satisfies the following conditions:

(a) the maximum energy deposition is in the central block (i.e., $E_{13}$ is the maximum);

(b) the energy deposition in the corner blocks is less than that in each of two neighboring blocks (i.e., $E_{7}<E_{8}$ and $E_{7}<E_{12}$, etc.);

(c) about $50 \%$ of shower energy must be deposited in the central row (and column) of cluster (i.e., $E_{12}+E_{13}+E_{14} \geq\left(E_{7}+E_{8}+E_{9}\right)+\left(E_{17}+\right.$ $\left.E_{18}+E_{19}\right)$, and $E_{8}+E_{13}+E_{18} \geq\left(E_{7}+E_{12}+\right.$ $\left.\left.E_{17}\right)+\left(E_{9}+E_{14}+E_{19}\right)\right)$.

\begin{tabular}{c|c|c|c|c|c|}
$\mathbf{1}$ & \multicolumn{1}{c}{$\mathbf{1}$} & \multicolumn{1}{c}{3} & \multicolumn{1}{c}{4} & \multicolumn{1}{c}{} \\
\cline { 2 - 6 } & $E_{1}$ & $E_{2}$ & $E_{3}$ & $E_{4}$ & $E_{5}$ \\
\cline { 2 - 6 } & $E_{6}$ & $E_{7}$ & $E_{8}$ & $E_{9}$ & $E_{10}$ \\
\cline { 2 - 6 } $\mathbf{4}$ & $E_{11}$ & $E_{12}$ & $E_{13}$ & $E_{14}$ & $E_{15}$ \\
\hline & $E_{16}$ & $E_{17}$ & $E_{18}$ & $E_{19}$ & $E_{20}$ \\
\cline { 2 - 6 } $\mathbf{5}$ & $E_{21}$ & $E_{22}$ & $E_{23}$ & $E_{24}$ & $E_{25}$ \\
\hline
\end{tabular}

Fig. 20. The cluster scheme with the labeling of the blocks location. A cluster is comprised of $3 \times 3$ central blocks.

In the case where the shower center is in the middle of the central block, $E_{13} \sim 84 \%$ of the total shower energy and about $14 \%$ of the total energy is in the four neighboring blocks, $E_{8}=E_{12}=E_{14}=E_{18}=3.5 \%$. The remaining $2 \%$ of the total shower energy is in the four corner blocks. Even at the largest luminosity used in the RCS experiment the probability to observe two clusters with energies above
$50 \%$ of the elastic value was less than $10 \%$, so for the 704 blocks hodoscope the two-cluster overlap was very unlikely. The shower energy reconstruction required a calibration of the calorimeter channels. The purpose of the calibration is to define the coefficients for transformation of the ADC amplitudes to energy deposition for each calorimeter module. I.e., define $C_{i}$ such that $E_{i}=C_{i} \cdot\left(A_{i}-P_{i}\right)$, where: $A_{i}$ is the ADC amplitude, $P_{i}$ is an amplitude pedestal, $C_{i}$ is a calibration coefficient, and $E_{i}$ is the energy deposited in the $i$-th block. The calibration constants were defined by minimizing the functional:

$\chi^{2}=\sum_{n=1}^{N}\left[\sum_{i \in M^{n}} C_{i} \cdot\left(A_{i}^{n}-P_{i}\right)-E_{e}^{n}\right]^{2}$

Here:

$n=1 \div N$ - number of the selected calibration event;

$i$ - number of the block, included in the cluster;

$M^{n}$ — set of the blocks' numbers, in the cluster;

$A_{i}^{n}$ - amplitude into the $i$-th block;

$P_{i}$ - pedestal of the $i$-th block;

$E_{e}^{n}$ - known energy of a particle;

$C_{i}$ - calibration coefficients, which need to be fitted.

The scattered electron energy $E_{e}^{n}$ was calculated by using the energy of the primary electron and the scattered electron angle. A cut on the proton momentum-angle correlation was used to select clean elastic events. Having the calculated calibration constants, the energy deposition $E$, and the $X, Y$ coordinates of the shower gravity center were calculated by the formulas:

$E=\sum_{i \in M} E_{i}, \quad X=\sum_{i \in M} E_{i} \cdot X_{i} / E, \quad Y=\sum_{i \in M} E_{i} \cdot Y_{i} / E$

where: $i$ - the number of calorimeter block, included in the cluster; $M$ - the set of blocks numbers, included in the cluster; $E_{i}$ - the energy deposition in the $i$-th block; $X_{i}, Y_{i}$ - the $X$ and $Y$ coordinates of the $i$-th block center. The resulting position resolution was found to be $7 \mathrm{~mm}$ for $2.3 \mathrm{GeV}$ electrons. The measurement of the shower center of gravity by using eqs. (1) is the simplest method to define the coordinates of high- energy photons and electrons in a hodoscope spectrometer. For the second test run [7] a two-coordinate MWPC was constructed. Its good position resolution of $1 \mathrm{~mm}$ allowed a direct investigation of the calorimeter position resolution. The chamber had 128 sensitive wires in each $\mathrm{X}$ and $\mathrm{Y}$ direction with a wire spacing of $2 \mathrm{~mm}$. The coordinates calculated by the gravity method were used for a more accurate estimate of the incident electron position in the calorimeter. For this iteration, the coordinate $x_{o}$ of the shower center of gravity inside the cell (i.e. relative the low bound of cell) was used. An estimate $x_{e}$ of the coordinate was presented in the form of a polynomial $P\left(x_{o}\right)$ :

$x_{e}=P\left(x_{o}\right)=a_{1} \cdot x_{o}+a_{3} \cdot x_{o}^{3}+a_{5} \cdot x_{o}^{5}+a_{7} \cdot x_{o}^{7}+a_{9} \cdot x_{o}^{9}$ 
For symmetry reasons, only odd degrees of the polynomial are present. The coefficients $a_{n}$ are calculated by minimizing the functional:

$\chi^{2}=\sum_{i=1}^{N}\left[P\left(a_{n}, x_{o}^{i}\right)-x_{t}^{i}\right]^{2}$.

where:

$i=1 \div N$ - number of event;

$x_{o}^{i}$ - coordinate of the shower center of gravity inside the cell;

$x_{t}^{i}$ - coordinate of the track (MWPC) on the calorimeter plane; ted.

$a_{n}$ - coordinate transformation coefficients to be fit-

The resulting resolution obtained from such a fit is about $5.5 \mathrm{~mm}$ for a scattered electron energy of $2.3 \mathrm{GeV}$. Figure 21 shows the correlation between the energy measured in the calorimeter and the scattering angle for the electrons elastically scattered from the protons.

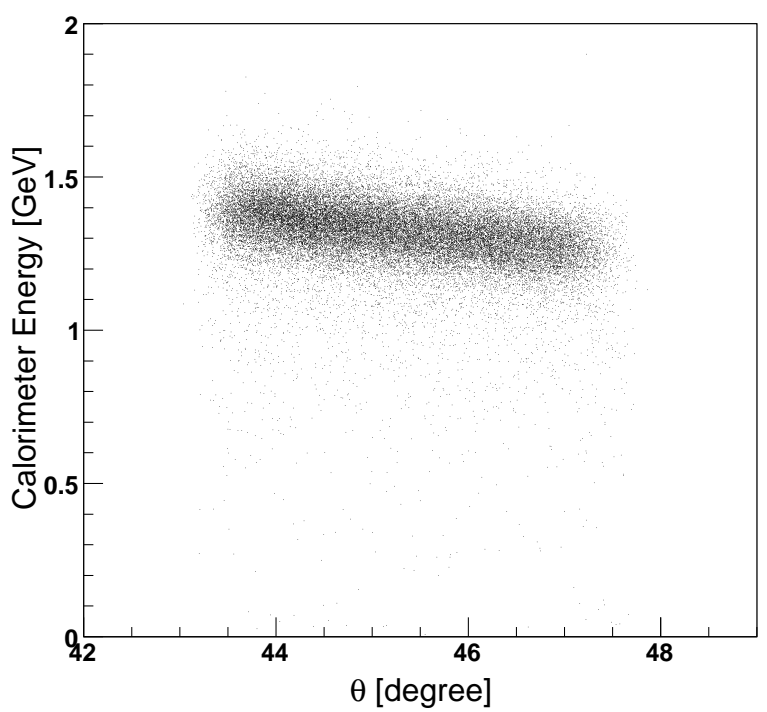

Fig. 21. The correlation between the energy measured by the calorimeter and the scattering angle for elastic $e-p$ scattering.

\subsection{Radiation Damage}

The energy resolution for the calorimeter obtained at the beginning of experiment E99-114 was 5.5\% (for a $1 \mathrm{GeV}$ photon energy) and had become $10 \%$ by the end of the experiment. The observed loss of energy resolution is due to radiation damage of the lead glass. The accumulated radiation dose was estimated to be $3-6 \mathrm{krad}$ by using the average width and the shift of the ADC pedestals. Because the radiator triples the radiation level, one can find that the observed radiation effects correspond to a 500 hours experiment with a $15 \mathrm{~cm}$ LH target and $50 \mu \mathrm{A}$ beam. The front face of the calorimeter during the experiment was protected by plastic material with an effective thickness of $10 \mathrm{~g} / \mathrm{cm}^{2}$. Most of the time the calorimeter was located at distance of $5-8 \mathrm{~m}$ at an angle of $40-50^{\circ}$ with respect to the electron beam direction.

The transparency of 20 lead-glass blocks was measured after the experiment (see Fig. 22). The plot presents a relative transmission through $4 \mathrm{~cm}$ of glass in the direction transverse to the block length at different locations. The values were normalized to the transmission through similar lead-glass blocks not used in the experiment. The transmission measurement was done with a blue light LED $\lambda_{\max }$ of $430 \mathrm{~nm}$ and Hamamatsu photo-diode 1226-44. A UV tech-

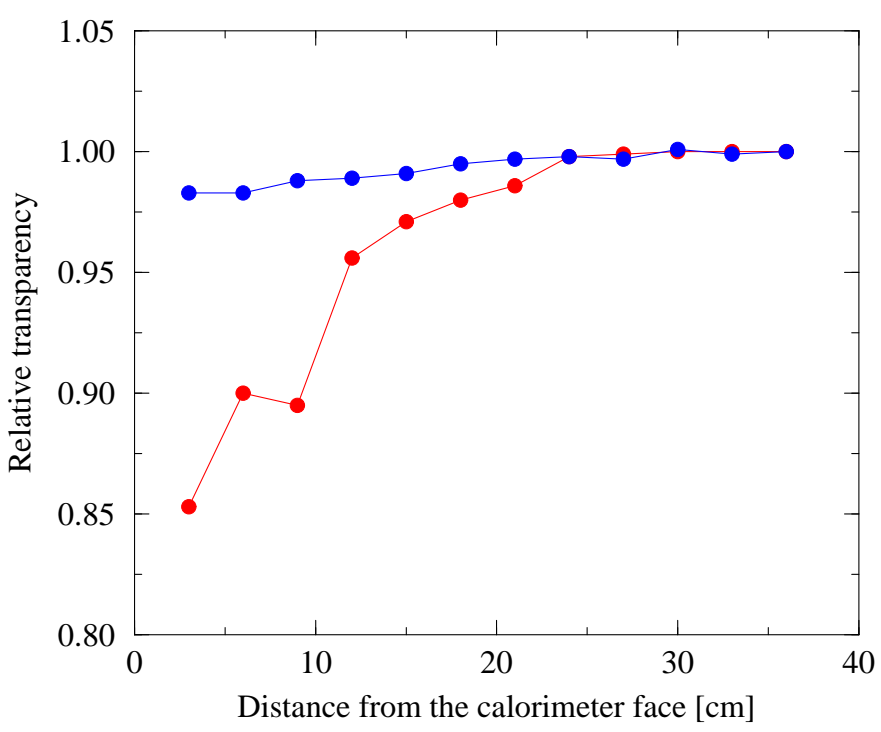

Fig. 22. The blue light attenuation in $4 \mathrm{~cm}$ of lead-glass vs distance from the front face of calorimeter measured before (red) and after (blue) UV irradiation.

nique was developed and used on the E99-114 calorimeter to cure radiation damage. The UV light was produced by a $10 \mathrm{~kW}$ total power 55-inch long lamp of type A94551FCB 7 . The lamp was installed vertically at a distance of 45 " from the calorimeter face. A quartz plate C55QUARTZ used as a filter of the infrared light. The intensity of the UV light at the face of the lead-glass blocks was found to be75 $\mathrm{mW} / \mathrm{cm}^{2}$ by using a UVX digital radiometer 8 . Irradiation by the UV light was done in 18 hours in situ without disassembling the lead-glass stock. All PMTs were removed before irradiation to insure the safety of the photocathode. We also successfully tested an alternative method to restore the lead-glass transparency in which lead-glass blocks were heated to about $250^{\circ} \mathrm{C}$ for several hours.

\section{Summary}

Here, we described the design and performance of the electromagnetic calorimeter used in the JLab RCS experiment. Good energy and position resolutions enable performance

\footnotetext{
7 Manufactured by American Ultraviolet, Lebanon, IN 46052, USA

8 Manufactured by UVP, Inc., Upland, CA 91786, USA
} 
of a high luminosity measurement of the RCS process in a wide range of kinematical parameters.

\section{Acknowledgments}

We acknowledge the RCS collaborators who helped to operate the detector and the JLab technical staff for providing outstanding support, and specially D. Hayes, T. Hartlove, T. Hunyady, and S. Mayilyan for help in the construction of the lead-glass modules. We appreciate S. Corneliussen's careful reading of the manuscript and his valuable suggestions. This work was supported in part by the National Science Foundation in grants for the University of Illinois University and by DOE contract DE-AC05-84ER40150 under which the Southeastern Universities Research Association (SURA) operates the Thomas Jefferson National Accelerator Facility for the United States Department of Energy.

\section{References}

[1] C. Hyde-Wright, A. Nathan, and B. Wojtsekhowski, spokespersons, JLab experiment E99-114.

[2] Charles Hyde-Wright and Kees de Jager Ann.Rev.Nucl.Part.Sci. 54, 217 (2004).

[3] A.V. Radyushkin, Phys. Rev. D58, 114008 (1998).

[4] H.W. Huang, P. Kroll, T. Morii, Eur. Phys. J. C23, 301 (2002); erratum ibid., C31, 279 (2003).

[5] R. Thompson, A. Pang, Ch.-R. Ji, Phys. Rev. D73, 054023 (2006).

[6] M.A. Shupe et al., Phys. Rev. D19, 1929 (1979).

[7] E. Chudakov et al., Study of Hall A Photon Spectrometer. Hall A internal report, 1998.

[8] J. Alcorn et al., Nucl. Instr. Meth. A 522, (2004) 294.

[9] D.J. Hamilton et al., Phys. Rev. Lett. 94, 242001 (2005), A. Danagoulian et al., Phys. Rev. Lett. 98, 152001 (2007).

[10] Yu.D. Prokoshkin et al., Nucl. Instr. Meth. A 248, 86102 (1986).

[11] M.Y. Balatz et al., Nucl. Instr. Meth. A 545, 114 (2005).

[12] V. Popov et al., Proceedings of IEEE 2001 Nuclear Science Symposium (NSS) And Medical Imaging Conference (MIC). Ed. J.D. Valentine IEEE (2001) p. 634-637; V. Popov, Nucl. Instr. Meth. A 505, 316 (2003).

[13] W.A. Watson et al., CODA: a scalable, distributed data acquisition system, in: Proceedings of the Real Time 1993 Conference, p. 296;

[14] E. Jastrzembski et al., The Jefferson Lab trigger supervisor system, 11th IEEE NPSS Real Time 1999 Conference, JLabTN-99-13, 1999.

[15] R.R. Crittenden et al., Nucl. Instr. Meth. A 387, 377 (1997). 